\title{
Escape FolRoom: Propuesta de juego integrada en clase de Formación y Orientación Laboral
}

\section{RESUMEN}

Durante la práctica docente se ha detectado una cierta desmotivación o frustración por parte del alumnado en el módulo de Formación y Orientación Laboral. Para combatir este problema y con la finalidad de alcanzar los objetivos propios de trabajar en equipo, como son colaborar en equipo aportando ideas y llegando a consensos, conocer el valor de los equipos de trabajo, diferenciar entre eficacia y eficiencia, identificar los distintos tipos de roles así como la figura de liderazgo y fomentar la interdependencia positiva y la exigibilidad personal, entre otros, se ha propuesto aplicar la metodología propia combinada de aprendizaje basado en juegos y gamificación por medio del diseño de un escape room educativo, juego que en la actualidad se encuentra bastante de moda y que combinan diversas características tales

\section{ABSTRACT}

Escape FolRoom: Proposal for an integrated game in the Training and Orientation class

During the teaching practice a certain demotivation or frustration has been detected by the students in the Training and Employment Orientation module. To combat this problem and in order to achieve the objectives of working in a team, such as collaborating in a team contributing ideas and reaching consensus, knowing the value of work teams, differentiating between effectiveness and efficiency, identifying the different types of roles as well as the figure of leadership and promoting positive interdependence and personal enforceability, among others, it has been proposed to apply the own combined methodology of game-based learning and gamification through the design of an educational escape room, a game that today It is quite fashionable 
como la diversión, la motivación y distintas herramientas de trabajo en equipo.

Finalmente, tras el análisis de las características y beneficios del presente recurso didáctico, se ha demostrado que el escape room es una herramienta idónea para enseñar a trabajar en equipo de una forma práctica y completa, pudiendo ser combinada con otras materias o temáticas adicionales.

Palabras Clave: Juegos, Aprendizaje basado en juegos, Trabajo en equipo, recurso educativo. and they combine various features such as fun, motivation and different teamwork tools.

Finally, after analyzing the characteristics and benefits of this teaching resource, it has been shown that the escape room is an ideal tool to teach teamwork in a practical and complete way, and can be combined with other additional subjects or themes.

Keywords: Games, Game-based learning, Teamwork, educational resource.

\section{Introducción}

Trabajar en equipo de forma idónea es una cualidad que en la actualidad se requiere bastante para todo tipo de experiencias laborales y que se caracteriza por una escasa formación previa en la misma. Por ello, qué mejor que comenzar a desarrollar estas cualidades desde edades tempranas o previas al mundo laboral.

Así mismo, para hacer divertida esta materia y aprender de forma más significativa se ha propuesto introducir como recurso didáctico el juego.

En primer lugar, se puede conceptualizar el juego como aquella actividad cuya fundamentación principal es obtener entretenimiento y diversión de aquella persona que lo ejecuta.

Jiménez (2003) alude a los juegos como actividades divertidas que requieren de una capacidad física y mental, que se realizan con satisfacción.

En determinadas ocasiones, el juego sirve de medio para evaluar los conocimientos previos de la persona, ayudando a obtener nuevas capacidades, destrezas y habilidades que son cruciales en su desarrollo social y personal (Rojas, 2009).

Tal y como describe Sutton-Smith (1978), el juego y todo aquello relacionado con el mismo son la clave de la educación integral, ya que para ponerlo en marcha se requiere de la interacción y de la actitud social. Siguiendo esta línea de pensamiento, apunta Gómez (2009), el juego ha desempeñado a lo largo de la historia un papel clave, no sólo por su componente lúdico, sino también como instrumento formador y a su vez propio de una manifestación cultural concreta. 
Los beneficios de educar por medio del recurso didáctico del juego son:

- Son actividades exitosas entre el alumnado, puesto que se conciben como actividades atractivas, creadoras y que fomentan la competencia. Este recurso favorece el proceso desarrollador del alumnado, llevando a cabo situaciones que potencian la empatía, el respeto, el trabajo en equipo y la aceptación de fracasos propios y reconocimiento de éxitos ajenos. (Chamoso, 2004).

- Requieren niveles de esfuerzo alto, tanto como de rigor, concentración y memoria, además de que es un instrumento fundamental para desarrollar la imaginación (Alsina, 2007).

- El juego es un instrumento idóneo para trabajar la diversidad (Contreras, 2004).

Concretamente, los juegos de escape room se componen por un número limitado de personas, las cuales son encerradas en una habitación decorada y ambientada en una temática determinada, con la finalidad de resolver un enigma final y así poder escapar de la misma antes de que transcurra el tiempo destinado para ello.

La primera evidencia del juego Escape Room actual, se encuentra en un tipo de videojuegos muy usados en la época de los ochenta, donde el propio jugador era el personaje del juego, ubicándolo en una habitación totalmente amueblada de acuerdo a una temática. Gran parte de sus características se remontan a juegos clásicos de mesa como "El Cluedo".

El primer Escape Room tal y como se conoce en la actualidad, tiene sus indicios en el año 2006 donde los ingenieros de Silicon Valey crearon el primer juego de Escape Room, basándose en novelas de la escritora Agatha Christie. Takao Kato, en el año 2008 creó un juego bastante similar al actual, pero con una principal diferencia, ya que la resolución de un reto o enigma final no era la clave principal del juego. En 2011 es el año en el que Attila Gyurkovics diseñó el primer escape room en la ciudad de Budapest, para así reutilizar edificios descuidados y algo abandonados para así darles otras funciones.

La clave del Escape Room se encuentra la Teoría del Flujo Csíkszentmihályi (Punset, 2008): que sea una meta clara, susceptible de alcanzarse, diseñada como un reto, necesaria retroalimentación, control de la situación y la percepción del tiempo se disipa con más facilidad.

Tal y como define en una entrada de su blog el docente Negre i Walcak (2018), también referida en la página web Educación 3.0 (2018) podemos concretar en diez razones las que nos conducen a utilizar un escape room educativo:

1. Fomenta la participación.

2. Se puede diseñar con cualquier tipo de contenido curricular.

3. Propicia la colaboración, cooperación y el trabajo en equipo. 
4. Fomenta la resolución de problemas.

5. Enriquece la comprensión lectora y verbal, por medio del intercambio de opiniones, de los diálogos entre ellos, etc.

6. Propone retos ante los que se requiere perseverancia.

7. Promueve un razonamiento crítico.

8. Adquieren la habilidad de trabajar bajo presión.

9. El alumnado es el protagonista de su propio aprendizaje.

10. Se aprende con diversión.

Por otro lado, por medio del uso de Escape Rooms Educativos el alumnado puede evolucionar en las aptitudes que se encuentran ligadas directamente al juego, o lo que también se hace llamar como "Gameful Attitudes". Este término se fundamenta en el libro "Reality is broken", reproduciendo textualmente a su autora McGonigal (2011, p.47)

Ser "gameful" significa traer las fuerzas psicológicas que naturalmente se muestran cuando se juegan a juegos, como son el optimismo, la creatividad, el coraje y la determinación a la vida real. Significa tener la curiosidad para jugar con diferentes estrategias para descubrir qué funciona mejor. Significa aumentar la resiliencia para enfrentar desafíos cada vez más difíciles con mayor y mayor éxito.

Un escape room es un juego destinado fundamentalmente a trabajar en equipo, a comunicarse y entenderse para que entre todos se descubran pistas y a través de las misma se resuelvan enigmas, actividades u otro tipo de pruebas con el reto final de escapar de la habitación en un tiempo determinado.

Por escape room educativos entendemos como aquel entorno educativo creativo, que puede orientarse para cualquier curso o nivel formativo y que hace uso de los componentes y características de un escape room tradicional, añadiendo contenidos, objetivos y elementos educativos concretos.

\section{Material y método}

Por un lado, se han utilizado al completo los recursos que componen en aula en la que se ha implementado el escape room, como son: las mesas y las sillas, los ordenadores, teclados y ratones, los armarios, las perchas, las ventanas y la puerta, la pizarra, la mesa y silla del docente, así como el ordenador, el cañón y su pantalla. 
Por otro lado, como material objeto de decoración, tematización, pruebas y pistas del escape room se han utilizado un código Qr, un puzle, varias cajas diversas, cofre, cadena, candados de numerosas clases, baraja de cartas, caja fuerte, traductor de signos egipcios, agenda, crucigrama, linterna, cápsulas, rotulador de tinta invisible, sopa de letras, mapas, caja mágica, cartulina, cinta adhesiva, rotuladores, bolígrafos, folios, pegamento, goma espuma, chinchetas, corcho, cinta aislante, adhesivos, sobres, noticias de periódicos, caja fuerte, lupa, espejo, caja laberinto, imanes, traductor de símbolos, etc.

En cuanto a la temporalización del mismo, el tiempo destinado para el ejercicio del juego es el de 60 minutos, por lo que se pretende que el alumnado resuelva el enigma final antes de transcurrir al completo dichos minutos. El tiempo será reflejado por medio de unos contadores diseñados específicamente para este juego.

No obstante, el cómputo del tiempo total no es ese exclusivamente, puesto que se precisa previamente formar los equipos, la asignación a cada uno de su escape room correspondiente, la explicación de qué es un escape room y cómo se juega, así como sus instrucciones, sus recomendaciones y sus prohibiciones, así como la visualización de vídeos introductorios para adentrar más en la temática del mismo.

Además, también se requiere de aproximadamente media hora para el montaje del mismo y un sinfín de horas previas para el diseño, puesta en marcha, horas extralaborales.

\section{Resultados}

Para llevar a cabo este escape room se decidió previamente que para que todo el alumnado al completo participara y crear un ambiente mayor de motivación, formar dos grupos, cada uno de ellos con cuatro miembros.

La temática dedicada a cada uno de estos dos escape rooms será:

- Prison-Fol.

- Cuenta atrás...3, 2, 1 \& FOL.

Se han orientado a temáticas distintas, enigmas y retos diferentes, para lo cual todos los miembros de ambos grupos deberán de participar conjuntamente para así poder alcanzar la última prueba que los hará libres y por ello mismo ganadores.

Este juego tiene como objetivo repasar y afianzar los contenidos de equipos de trabajo previamente explicados y estudiados, considerándolo como una herramienta o instrumento bastante innovador y útil 
para que el alumnado interiorice los contenidos previamente explicados y creen una reflexión crítica de trabajar de forma colaborativa.

Estos juegos se han diseñado partiendo de la base de la siguiente estructura sólida relativa a la consecución de las pruebas:

1. Para potenciar la colaboración y al mismo tiempo la diversidad dentro de los equipos, hemos construido pruebas donde sean aplicables distintas clases de habilidades, pudiendo ser tanto físicas, como de ingenio, manuales, de matemáticas o incluso de mera observación o de practicar la escucha activa. De esta forma todos los componentes del equipo que cuenten con distinto tipo de inteligencias, podrán aportar dichas habilidades que poseen, siendo todas ellas útiles y desarrollando así el sentido de pertenencia al equipo y poder llevar a una automotivación y poder así incluso incrementar su nivel de valor propio.

2. Para que se pueda trabajar en equipo de forma eficiente, se deberán de asignar roles en el mismo. Pues bien, previamente al comienzo le asignamos los roles a todos los miembros del equipo según las características de la personalidad del alumno/a como de sus habilidades más desarrolladas. Estos roles son: el de matemático (el que tenga que realizar los cálculos matemáticos), informático (el que use el móvil o el ordenador), cerrajero (el que introduzca las coordenadas o los códigos en el candado), lector o portavoz (el que lea en voz alta las pruebas y las pistas, y se dirija si así es preciso a la profesora), recolector (el que recolecte todas las pruebas que se vayan superando), asuntero (el que abra los cajones, levante objetos, inspeccione debajo de la mesa, etc. para así conseguir nuevas pistas) y el líder (será el que deba de guiar y coordinar el equipo, pudiendo elegir un tipo de líder de los ya aprendidos anteriormente).

3. Se le dará la opción de que los roles sean rotativos, es decir que cada veinte minutos han de cambiar de rol que obtienen y así trabajar de forma más dinámica y asumir y comprender más roles que puede ocupar en un equipo.

Una de las cajas que contiene una nueva pista se encuentra cerrada con un aspa de control que contiene tantos candados como miembros hay en el equipo, por lo que hasta que no se abran todos los candados, la caja no podrá abrirse completamente y no se obtendrá la pista. Así se aplicará en la práctica la interdependencia positiva y la exigibilidad personal.

4. Cuando se llegue a la mitad del tiempo, se le proporcionará por parte del docente un comodín. Para ello será necesario aplicar la técnica del puzle de Aroson. Así los "especialistas" en este caso los que ocupen cada rol de los dos equipos, es decir: todos los miembros que sean cerrajeros de los dos equipos deberán de sentarse juntos, y así igual con el resto de roles, y recibirán un comodín referente al cargo que desempeñen explicado por el profesor. Posteriormente estos miembros deberán de volver a su equipo base y explicar el comodín obtenido. 
5. Cada equipo tiene una libreta cerrada con un candado cuya llave se encuentra escondida. En dicha libreta hay una pista escrita con una tinta invisible, que sólo podrá reflejarse utilizando una linterna de luz ultravioleta. Sólo hay una para los dos equipos, por lo que será imprescindible que compartan la herramienta de forma adecuada y generosa para poder continuar con el juego.

6. Se han escondido todas las piezas de un puzle en diferentes lugares del aula. Deberán los participantes de cada equipo unir todas las piezas para poder descifrar un mensaje y así poder proseguir con la prueba.

7. Varias de las pistas se encuentran cerradas con candados, que previamente deberán de resolver el código por medio de una sopa de letras y un crucigrama.

8. Lo más importante es que el alumnado aplique dinámicas de trabajo en equipo, que sepan poner en práctica lo aprendido y que detecte los beneficios de ello aplicándolos por ellos mismos. No se trata únicamente de abrir candados, cajas o conseguir pistas, sino que trabajen verdaderamente en equipo, que negocien si es necesario, que se comuniquen de forma no violenta y que se ayuden los unos a los otros para poder así conseguir liberarse.

Para el diseño de esta actividad se ha partido de los elementos fundamentales anteriormente mencionados que componen la estructura de un escape room.

Tras una investigación exhaustiva, se concluye que es fundamental usar uno de los elementos que envuelve el desarrollo de la actividad de escape room, es decir una narrativa sólida sobre la cual se desarrollen retos.

Hemos diseñado las siguientes narrativas para dar un valor extra a nuestro trabajo:

\section{- Narrativa Prison-Fol:}

"Ha sido encarcelado el más prestigioso técnico de FOL del mundo, John Smith, ya que uno de sus principales rivales, Mr. White, movido por el odio y la envidia ha conseguido imputarle un delito de propiedad intelectual.

Al ser uno de los más virtuosos sabios en la actualidad y gracias a sus elevados conocimientos en la materia ha conseguido escapar de la celda en la que se encontraba preso.

Su paradero es desconocido, por ello necesitemos que averigüéis, vosotros, sus discípulos más aventajados en la materia de FOL, cómo ha escapado y para así saber cuál es su paradero actual y poder demostrar que es inocente.

El futuro de John Smith está en vuestras manos, FOL depende de vosotros”. 


\section{- Narrativa de Cuenta atrás...3, 2, 1 \& Fol:}

"Una bomba nuclear está programada para que explote dentro de 60 minutos.

Si explota la tierra será desbastada completamente, no sobrevivirá ser viviente alguno. El creador de la bomba, Mike Castle, es un experto en la materia de Formación y Orientación Laboral ya que ha trabajo durante mucho tiempo en el departamento de FOL de la NASA y tiene conocimientos muy técnicos sobre la materia, habiendo elaborado la bomba sobre los cimientos de esos conocimientos.

Se os ha encargado a vosotros, los más aventajados alumnos del Instituto Universal de FOL la operación más importante de vuestras vidas: ¡Salvar al planeta de la destrucción masiva! Nuestra esperanza radica únicamente en vosotros. Aplicar todos vuestros conocimientos de trabajo en equipo y habilidades para ello, nuestras vidas están en vuestras manos".

Por otro lado, desde el punto de vista de la estructura o patrón, es conveniente advertir de que existen diferentes tipos de patrones para el diseño de un escape room: lineal, multilineal y abierto.

En esta propuesta vamos a combinar dos de los patrones, por un lado, el lineal aplicado cuando cada equipo persiga una secuencia ordenada de retos; y, por otro lado, el multilineal cuando los equipos se encuentren en uno de los retos resolviendo la misma pista.

Desde el punto de vista de los retos, cada equipo tendrá al menos 10 retos que superar y todos los equipos tendrá un reto de cada tipo (los resultados/soluciones son distintos para cada equipo, aunque el tipo de enigma también puede ser diferente en algunas ocasiones y el orden en que se encuentran y ejecutan los retos también difiere entre los equipos).

Se pretende que todos los equipos puedan desarrollar las habilidades relativas a todos los tipos de reto seleccionados, lograr la totalidad de los objetivos propuestos y demostrar los conocimientos que se revisan con la actividad.

\section{PRISON-FOL:}

\section{Reto 1: De Introducción.}

El Escape room comienza con un vídeo introductorio para poner al equipo en situación y hacer que se adentren mejor en la dinámica y narrativa del juego.

\section{Reto 2: De Localización.}

Este reto es el inicial, una nota que recibe el equipo en un sobre y los dirige a una zona del aula: "Por donde entra el sol encontraréis vuestra misión". 


\section{Reto 3: Código Qr.}

En una ventana se encuentra pegado con celo una cartulina con un código Qr. En la cara opuesta de dicha cartulina encontrarán la siguiente pista: "Para superar esta prueba has de pedir el móvil a la carcelera”. Con este tipo de reto se trabaja la competencia digital, además de las competencias intrínsecas al juego que anteriormente hemos señalado y que en el próximo apartado desarrollaremos. Una vez escaneado el código se reflejará un mensaje: “¡Socorro! Soy John Smith y durante mi tiempo en prisión me vi obligado a llevar a cabo un plan para escapar de aquí, ya que mi vida corría un serio peligro. Mr. White, mi mayor rival me ha acusado de un delito que no he cometido y quiere acabar conmigo. $\mathrm{He}$ construido un pasadizo secreto que une mi celda con el exterior. No puedo deciros mucho más, no tengo tiempo. Esto es un rompecabezas. La clave para escapar está en superar las disfunciones en el equipo”. Si se interpreta correctamente el mensaje le llevará a buscar un rompecabezas, un puzle.

\section{Reto 4: Puzle.}

Situada sobre una mesa, hemos dispuesto una caja de color rosa sin cerrar con candado, donde se guardan distintas piezas de un puzle. Una vez resuelto el puzle se dilucidará una imagen con un preso y su número de condena, en el cual se encuentran subrayados los números: 2, 3 y 4.

\section{Reto 5: Cartas sobre la mesa.}

La combinación anteriormente descubierta abre el candado de un cofre que se encuentra encadenado. Este cofre custodia en su interior dos notas cuyo contenido es: "Tengo claro únicamente dos cosas: 1. Quién ha sido la persona que me ha traicionado. 2. Cuál es mi estrategia para escapar, mi as que he guardado bajo la manga", "No dejes pasar ningún detalle por muy pequeño que sea". Además, también custodia dos cartas: As de rombos (en esa carta se encuentra escrito del revés la siguiente pista: Estudiar inglés) y Joker (en esta carta se encuentra escrito muy pequeño la siguiente combinación: 00 0). Para resolver esta prueba han de utilizar el espejo y la lupa que se encuentran escondidos en sitios distintos.

\section{Reto 6: Biblioteca.}

En la prisión John le dedicaba mucho tiempo al estudio por lo que pudo en su celda crear una pequeña biblioteca, donde entre muchos libros se encontrará un diccionario de inglés que verdaderamente es una caja fuerte (cuya combinación es 00 0). En su interior encontrarán una frase escrita en el jeroglífico egipcio. El traductor que les ayudará a conseguir descifrar la pista se encuentra escondido en un cajón entre muchos papeles. Su traducción es: "El día de mi 40 cumpleaños hallarás la pista". 


\section{Reto 7: Enigma 1}

Se trata de un reto tanto de memoria como de investigación. La pista anterior les debe de conducir a una agenda que se encuentra escondida y buscar el día de su 40 cumpleaños, concretamente el día 12 de marzo, donde deberán de averiguar que con las cifras 12/3/79 hacen un código numérico: 12379. En dicha página se encuentra escrito a boli las letras: TEECG. Posteriormente deberán de buscar un crucigrama que se encuentra escondido próximo a la agenda. Las letras corresponden con las iniciales de cada palabra que se encuentra numerada, averiguando así que los números corresponden con las letras $(\mathrm{T} \rightarrow 1, \mathrm{E} \rightarrow 2, \mathrm{E} \rightarrow 3, \mathrm{C} \rightarrow 7, \mathrm{G} \rightarrow 9)$.

\section{Reto 8: Enigma 2}

El código anterior abrirá un candado de números presionados que cierra una caja transparente con muchos departamentos. En los distintos departamentos encontrarán: una linterna, material de decoración, una nota (Creo que me están envenenando la sopa) y varias cápsulas. En cada píldora nos encontraremos palabras que juntas forman la frase "Lo esencial es invisible a los ojos", para que así hagan uso de la linterna, la cual lleva pegada una indicación de "úsame".

\section{Reto 9: Enigma 3}

La anterior pista les conducirá a una sopa de letras que se encuentra sobre una mesa, donde con tinta invisible se encuentra oculta la frase: "Encuentra el mapa y encontrarás el túnel".

\section{Reto 10: Logro}

Colgado en la pared, sin ver a primera vista, se encuentran muchos y diversos mapas tanto de la zona donde se encuentra ubicada la prisión, así como mapas internos de la misma. En uno de esos mapas se encuentra señalado de color rojo una zona que coincidiendo con la ubicación paralela del aula corresponde a la mesa del docente.

Una vez ubicados en la mesa de la profesora, en el cajón se encuentra escondida una caja mágica de madera con la etiqueta: "Ábreme". En su interior se encuentra la pista final: "Ninguno de nosotros es tan bueno como todos nosotros juntos", pista que deberán de introducir en el contador diseñado expresamente para este escape y que se encuentra operativo en un ordenador, donde introduciéndola correctamente el contador parará y habrán ganado el juego siempre y cuando no haya finalizado los 60 minutos de duración. 


\section{CUENTA ATRÁS....3, 2, 1 \& FOL:}

\section{Reto 1: De Introducción}

El Escape room comienza con un vídeo introductorio para poner al equipo en situación y hacer que se adentren mejor en la dinámica y narrativa del juego.

\section{Reto 2: De Localización}

Este reto es el inicial, una nota que recibe el equipo en un sobre y los dirige a una zona del aula: "Por donde entráis a la habitación encontraréis vuestra misión".

\section{Reto 3: Noticia de periódico}

En la puerta del aula se encuentra pegada con celofán una noticia de periódico diseñada expresamente para este escape room, en la cual se encuentra oculta la pista: "La clave está en la planificación del trabajo en equipo".

\section{Reto 4: Enigma 1}

Dicha pista les deberá dirigirse hacia la agenda. Justamente en la página correspondiente a la fecha de la anterior noticia (6 de agosto) se encuentran escritas a bolígrafo las siglas: RGFT 0. Próximo a dicha agenda se encuentra escondido un crucigrama. Se deberá de completar el crucigrama con los contenidos en la materia y así llegar a la conclusión de que las iniciales trascritas en la página corresponden con los números, de la siguiente forma: Rà5, Gà6, Fà8, Tà9, $0 \rightarrow 0$.

\section{Reto 5: Enigma 2}

El código numérico anterior es la clave que abre el candado que cierra una caja transparente con múltiples apartados. En su interior se encuentran ocultas tres tipos de pistas: 1. La linterna de luz ultravioleta. 2. Las píldoras. 3. Unas llaves. En el interior de las píldoras se encuentran escondidas varias palabras que agrupadas forman la frase: "Lo esencial es invisible a los ojos". Junto a la linterna se ubica una etiqueta que pone: "úsame, y junto a las llaves una nota que expresa: Estas llaves abren lo guardado con máxima seguridad”.

\section{Reto 6: Las cartas sobre la mesa}

La pista que se encuentra próxima a las llaves deberá de conducirles hacia una caja fuerte escondida en una de las rejillas de las mesas de los estudiantes, la cual guarda con máxima seguridad 2 cartas: 
El As de picas: donde se encuentra escrito con tinta invisible la frase "Sin lupa no podrás ver". Deberán de hacer uso de la linterna anteriormente encontrada.

El rey de corazones: donde se encuentran dibujados pequeños símbolos que son idénticos a los que contiene otra caja, necesitando para verlos bien una lupa.

\section{Reto 7: Caja laberinto}

La caja laberinto se trata de una caja forrada con símbolos antiguos que por medio de unos imanes custodia unas llaves. Para ello se ha de encontrar la salida correcta del laberinto que descubrirá dichas llaves. Deben de encontrar un traductor de los símbolos, donde se oculta la palabra trabajo en equipo.

\section{Reto 8: Enigma 3}

Estas llaves son las que abren una caja de madera cerrada con una cadena que a su vez se encuentra cerrada con un candado y que oculta una pista: "Durante un año me dediqué a investigar todas las noticias sobre la bomba nuclear”.

\section{Reto 9: Prensa}

En un corcho que se encuentra colgado de la pared, se encuentran colgadas numerosas noticias de periódicos en distintos idiomas sobre la bomba nuclear. Hay palabras que tienen números en la parte interior de la misma, números del 1 al 21. En orden cada palabra forma la frase: "Mira en la caja de madera".

\section{Reto 10: Logro}

Deberán de encontrar una caja de madera que se encuentra escondida bastante bien y conseguir averiguar cómo se abre. En su interior se encierra la pista que contiene la clave final: "Si quieres llegar rápido camina solo, si quieres llegar lejos camina en equipo". Esa frase es la clave que han de introducir en el contador habilitado en un ordenador (diseñado expresamente para esta prueba) y que parará el contador y así podrán abrir la puerta que hace que salgan de la habitación antes de culminar los 60 minutos.

\section{Conclusiones}

De los resultados obtenidos, se puede destacar que el presente juego, escape folroom, es un recurso didáctico bastante útil y que produce un acercamiento práctico de materias que en esencia son puramente teóricas. Este hecho provoca que en el alumnado crezca el interés y la dedicación por su estudio, ya que además se divierten aprendiendo. 
Los resultados obtenidos han sido analizados en base a las opiniones e inquietudes del alumnado participante. Los aspectos valorados fueron por un lado los correspondientes al propios escape room, como son su organización, su dinámica, su composición y su decoración, y por otro lado, los contenidos objeto del juego, como son las cualidades de trabajar en equipo, la motivación y la interiorización de los contenidos aprendidos.

Las variables utilizadas para la valoración han sido: la resolución de pistas, la motivación, el desarrollo de la capacidad crítica, la obtención de un aprendizaje más significativo, el diseño del escape room y el trabajo en equipo. En general, la valoración ha sido bastante positiva en todos sus aspectos.

Desde el punto de vista de la motivación se ha demostrado que aprender utilizando un recurso gamificado como el escape room, hace crecer en gran medida la motivación del alumnado por parte de la materia que aprende.

Por otra parte, desde la base del desarrollo de la capacidad crítica se ha analizado y verificado que esta actividad fomenta el pensamiento crítico entendido desde el punto de vista aportado por Ennis (1985) ), que lo define como un pensamiento racional y reflexivo de una persona que le conlleva a tomar una determinada decisión de qué hacer o creer, tratándose de un tipo de pensamiento que caracteriza a la persona como un ser humano racional y que además tiene como objetivo actuar, surgiendo en la mayoría de los casos en las resoluciones de problemas o en las relaciones con otras personas.

Pues bien, este desarrollo del pensamiento crítico o la habilidad de resolver problemas se manifiesta en las partes de en las dificultades o impedimentos a la hora de superar los retos, superar las disfunciones de los equipos, seleccionar entre lo verídico y lo falso, entre lo relevante y lo secundario, entre las verdaderas pistas y aquellas para despistar.

Desde la posición de guía, hemos podido observar como el alumnado ha transferido conocimiento entre sí, llegando a concluir con estrategias muy válidas para solucionar los retos.

A su vez, se ha demostrado que el alumnado ha obtenido un aprendizaje más significativo, Partiendo de la base del concepto que porta Bruner (1963) referente a este punto, hay que resaltar la premisa de que al alumnado siempre se le debe de proporcionar con el aprendizaje un sentimiento de entusiasmo que acompañe al aprendizaje. Se debe de convertir al alumnado en el propio y principal protagonista de su aprendizaje, pariendo de la base de los conocimientos previos obtenidos y en sus gustos, consiguiendo así alcanzar un aprendizaje significativo.

En cuanto a los contenidos asimilados de trabajo en equipo, el principal objetivo era el de aplicar determinados retos y pruebas enfocadas a actividades enmarcadas en el contexto de Formación Profe- 
sional de Grado Medio, en el módulo de Formación y Orientación Laboral, por medio del diseño de un escape room educativo en el aula. El alumnado con el que se ha trabajado no está acostumbrado a trabajar en equipo, ni tampoco a obtener un aprendizaje por medio de recursos innovadores y que se desvinculan un poco de las clases tradicionales. Además, esta actividad se complementa con el factor diversión, lo que provoca que los contenidos sean aprendidos y asimilados de forma más significativa.

Una vez expuestos los resultados y analizados previamente, valorando esencialmente las opiniones del alumnado participante, podemos verificar la aceptación y éxito de lo aprendido relacionado con la metodología aplicada, concretamente con la actividad de escape room educativo. Es importante no dejar de lado, el alto nivel de contestaciones positivas a la pregunta de si volverían a repetir la experiencia en clase, que nos hace pensar que es una actividad que ha agradado a la mayoría del alumnado, potenciando sus intereses, habilidades y competencias.

Por otra parte, desde el punto de vista de la implementación de la experiencia del breakout requirió mucho trabajo y esfuerzo a la hora de su organización y diseño, que desde nuestro punto de vista sería el único inconveniente de esta actividad que para que tenga éxito y se diseñe bien se requiere mucho tiempo de diseño y de montaje, sin tener en cuenta el coste añadido de la adquisición de los materiales que sirve de componentes del mismo. No obstante, como ya hemos dicho, los resultados fueron bastante satisfactorios lo que provoca que ese esfuerzo tenga su merecida recompensa.

Como propuesta de mejora para futuros diseños de escape rooms educativos, se propone realizarlo con contenidos interdisciplinares, es decir, contenidos de materia de formación y orientación laboral y de otras disciplinas. También es interesante proponer crear uno que trabaje varias Unidades de Trabajo o Unidades Didácticas, actividad a modo de resumen y para afianzar contenido.

\section{Referencias}

Alsina, C. (2007). Educación matemática e imaginación. Madrid: Unión.

Bruner, J. S. (1963). El proceso de la educación. México: UTEHA.

Chamoso, J. M.; Durán, J. García, J. Martín; J. Rodríguez, M. (2004): Análisis y experimentación de juegos como instrumentos para enseñar matemáticas. Madrid: Suma.

Contreras, M. (2004). Las matemáticas de ESO y Bachillerato a través de los juegos. Madrid: Unión. Recuperado el 08/04/2013: http://www.mauriciocontreras.es/JUEGOSM.htm 
EDUCACIÓN 3.0 (15 de agosto, 2018). 10 razones para utilizar un escape room educativo en clase. Recuperado de: https://www.educaciontrespuntocero.com/noticias/razones-escaperoom-educativo/78689.html

EDUCACIÓN 3.0. (1 de junio, 2018). Recopilamos Las mejores experiencias con sala de escape educativo. Recuperado de https://www.educaciontrespuntocero.com/experiencias/ experiencias-con-escape-room-educativos/87179.html

Ennis, R. H. (1985). A logical basis for measuring critical thinking skills. London: Educational Leadership, 43(2), pp. 44-48.

Gómez, A. M. (2009). La importancia del juego y desarrollo en Educación Infantil. Cuadernos de Educación y desarrollo, 1(10). Barcelona: Altamar. Recuperado de http://www.eumed. net/rev/ced/10/amgg.htm

Jiménez, R. (2003): Aprender matemáticas jugando. Madrid: Alianza. Recuperado el 8 de abril de 2013: http://www.juntadeandalucia.es/averroes/ cepco3/competencias/mates/secundaria/premio aprende matematicas jugando.pdf

McGonigal, J. (2011). SuperBetter: The Power of Living Gamefully. New York: Penguin Books: Reprint.

Negre i W. C. (2018). 'BreakoutEdu', microgamificación y aprendizaje significativo. Recuperado el 26 de julio de 2017: https://www.educaweb.com/noticia/2017/07/26/breakoutedumicrogamificacion-aprendizaje-significativo-15068/

Punset, E. (2008, octubre 5). La felicidad es un 'estado de flujo' [Entrada de blog]. Recuperado de: https://www.eduardpunset.es/418-charlas-con-la-felicidad-es-un-estado-de-flujo/

Rojas, I. R. (2009): Aplicación de juegos lógicos en Juventud Salesiana. Madrid: Unión, 19, 150-156.

Sutton-Smith, B . (1978). Die dialektik des spiels. Deutschland: Schondorf. 
